Recent progress in the quantitative validation of JOREK simulations of ELMs in JET

This content has been downloaded from IOPscience. Please scroll down to see the full text.

2017 Nucl. Fusion 57076006

(http://iopscience.iop.org/0029-5515/57/7/076006)

View the table of contents for this issue, or go to the journal homepage for more

Download details:

IP Address: 207.162.240.147

This content was downloaded on 16/05/2017 at 13:36

Please note that terms and conditions apply. 


\title{
Recent progress in the quantitative validation of JOREK simulations of ELMs in JET
}

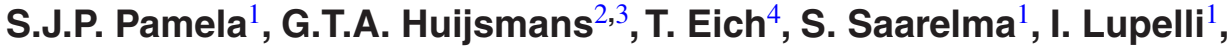 \\ C.F. Maggi ${ }^{1}$, C. Giroud ${ }^{1}$, I.T. Chapman ${ }^{1}$, S.F. Smith ${ }^{1,5}$, L. Frassinetti ${ }^{6}$, \\ M. Becoulet ${ }^{2}$, M. Hoelzl ${ }^{4}$, F. Orain ${ }^{4}$, S. Futatani ${ }^{7}$ and JET Contributors ${ }^{a}$ \\ EUROfusion Consortium, JET, Culham Science Centre, Abingdon, OX14 3DB, United Kingdom \\ 1 CCFE, Culham Science Centre, Abingdon, Oxon, OX14 3DB, United Kingdom \\ 2 CEA, IRFM, F-13108 Saint-Paul-lez-Durance, France \\ ${ }^{3}$ Eindhoven University of Technology, Eindhoven, Netherlands \\ ${ }^{4}$ Max-Planck-Institut für Plasmaphysik, Boltzmannstrasse 2, D-85748 Garching bei München, Germany \\ 5 Department of Physics, York Plasma Institute, University of York, Heslington, York YO10 5DD, \\ United Kingdom \\ ${ }^{6}$ Division of Fusion Plasma Physics, KTH Royal Institute of Technology, Stockholm SE, Sweden \\ 7 CASE Department, Barcelona Supercomputing Center, Barcelona, Spain \\ E-mail: stanislas.pamela@ukaea.uk
}

Received 19 December 2016, revised 31 March 2017

Accepted for publication 20 April 2017

Published 16 May 2017

\begin{abstract}
Future devices like JT-60SA, ITER and DEMO require quantitative predictions of pedestal density and temperature levels, as well as inter-ELM and ELM divertor heat fluxes, in order to improve global confinement capabilities while preventing divertor erosion/melting in the planning of future experiments. Such predictions can be obtained from dedicated pedestal models like EPED, and from non-linear MHD codes like JOREK, for which systematic validation against current experiments is necessary. In this paper, we show progress in the quantitative validation of the JOREK code using JET simulations. Results analyse the impact of diamagnetic terms on the dynamics and size of the ELMs, and evidence is provided that the onset of type-I ELMs is not governed by linear MHD stability alone, but that a nonlinear threshold could be responsible for large MHD events at the plasma edge.
\end{abstract}

Keywords: JET-ILW, ELM, pedestal, MHD, peeling ballooning modes, JOREK, simulations

(Some figures may appear in colour only in the online journal)

\section{Introduction and motivation}

Elaborate experimental scaling laws for the ITER tungsten divertor provide an estimate of the ELM (Edge-LocalisedMode) and inter-ELM target heat fluxes [1,2]. These predictions can be reinforced by numerical simulations of large-scale instabilities, like peeling-ballooning (PB) modes, to describe the characteristic dynamics of ELMs, as well as small-scale turbulence of kinetic-ballooning modes (KBMs)

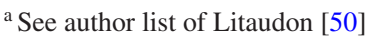

and ion-temperature-gradient (ITG) instabilities to describe the cross-field transport in the pedestal which regulates the plasma and energy exhaust across the separatrix in inter-ELM regimes.

Several nonlinear MHD codes, such as JOREK, BOUT ++ , HESEL and EMEDGE3D in Europe [3-8], M3D-C1 and NIMROD in the US [9, 10], or MEGA in Japan [11-13], can obtain advanced ELM simulations, with challenging physics effects like bi-fluid diamagnetic rotation and current, low resistivity and viscosity levels, as well as high poloidal/ toroidal resolutions. In the last decade, nonlinear MHD codes 
have focused their efforts on obtaining qualitative agreement with experimental observations, by considering key ELM characteristics like the formation of hot plasma filaments that are ejected through the separatrix, the collapse of the pedestal pressure, and the transport of energy to the divertor and first wall components. Recent increases in computational resources available to fusion research are now enabling the start of quantitative comparisons with experiments, which is the compulsory path towards predictions for future devices like ITER. In sight of the urgent need for such predictions, this paper discusses the latest progress of quantitative validations for the nonlinear MHD code JOREK against experimental data from the JET-ILW device.

In particular, one of the main issues for all nonlinear MHD codes that address ELM physics is the methodology used for the simulation set-up, and more precisely the initial boundary conditions. Unless multiple ELM cycles are simulated, the initial condition is typically chosen to be an unstable axisymmetric equilibrium with small toroidal perturbations (of the order of numerical noise). This paper will show that, if these initial conditions are used, nonlinear coupling cannot be obtained in the critical phase of the ELM crash; it will only occur in the late phase of the ELM, at which point it is too late to make a significant difference in ELM dynamics.

The paper is organised as follows. In section 2, we provide details on the JOREK code, including the physics model used for the simulations, and how JET experimental data is used to create initial conditions for the simulations. In section 3, we describe the results on the quantitative validation of JOREK simulations for ELMs in JET, and how these are affected by diamagnetic terms. Section 3 will also discuss the nonlinear aspect of simulations and the role played by initial value conditions concerning coupling between toroidal modes. In the conclusion, section 4 , we discuss how predictions for future devices with the JOREK code need to rely on multi-cylce ELM simulations, and what this implies for future simulations.

\section{The JOREK code}

\subsection{The physics and numerical models}

The 3D nonlinear MHD code JOREK was developed by Huysmans et al with the specific aim to produce simulations of edge-localised-modes [3, 4]. The MHD model used for the present paper is similar to that used in previous ELM studies [14-18]. It is a five-field reduced MHD model for the variables $\psi$ (poloidal magnetic flux), $\Phi$ (electric potential), $\overrightarrow{\mathrm{V}}_{\|}$(parallel velocity), $\rho$ (density), $T$ (total temperature), including the two-fluid diamagnetic effects. The reduction of the equations assumes that the perpendicular velocity lies in the poloidal plane, and that the toroidal magnetic field is constant in time, so that the total plasma velocity and the total magnetic field are expressed respectively as

$$
\begin{aligned}
\overrightarrow{\mathrm{v}}_{\mathrm{tot}} & =\overrightarrow{\mathrm{v}}_{\|}+\overrightarrow{\mathrm{v}}_{\perp}=\overrightarrow{\mathrm{v}}_{\|}+\overrightarrow{\mathrm{v}}_{E}+\overrightarrow{\mathrm{v}}_{* i} \\
& =v_{\|} \vec{B}+R \vec{e}_{\phi} \times \nabla \Phi+\frac{\delta^{*} R}{\rho} \vec{e}_{\phi} \times \nabla p_{i},
\end{aligned}
$$

$$
\vec{B}=\vec{B}_{\phi}+\vec{B}_{p}=\frac{F_{o}}{R} \vec{e}_{\phi}+\frac{1}{R} \nabla \psi \times \vec{e}_{\phi},
$$

where $R$ is the major radius, $\vec{e}_{\phi}$ is the toroidal unit vector and $F_{o}=B_{o} R_{o}$, with $B_{o}$ being the magnetic field amplitude at the reference major radius $R=R_{o}$. The diamagnetic component of the perpendicular velocity is represented by the third term $\overrightarrow{\mathrm{V}}_{* i}=\delta^{*} R \rho^{-1} \overrightarrow{\boldsymbol{e}}_{\phi} \times \nabla p_{i}$, where $p_{i}$ is the ion pressure and $\delta^{*}=\left(\Omega_{c i} R_{o}\right)^{-1}$, with the ion gyrofrequency $\Omega_{c i}=e B_{o} / m_{i}$. Substituting the identities (1) and (2) into the visco-resistive MHD equations gives the reduced MHD model, first derived by Strauss [19], with two equations for the parallel and the perpendicular momentum,

$$
\begin{gathered}
\rho \frac{\mathrm{d} \overrightarrow{\mathrm{v}}_{E}}{\mathrm{~d} t}=-\rho \overrightarrow{\mathrm{v}}_{* i} \cdot \nabla \overrightarrow{\mathrm{v}}_{E}-\nabla_{\perp} p+\vec{J} \times \vec{B} \\
+\mu \nabla^{2}\left(\overrightarrow{\mathrm{v}}_{E}+\overrightarrow{\mathrm{v}}_{* i}\right)+\mu_{\mathrm{hyp}} \nabla^{4}\left(\overrightarrow{\mathrm{v}}_{E}+\overrightarrow{\mathrm{v}}_{* i}\right) \\
\rho \frac{\mathrm{d} \overrightarrow{\mathrm{v}}_{\|}}{\mathrm{d} t}=-\rho \overrightarrow{\mathrm{v}}_{\|} \cdot \nabla \overrightarrow{\mathrm{v}}_{\|}-\nabla_{\|} p+\mu \nabla^{2} \overrightarrow{\mathrm{v}}_{\|}+\mu_{\mathrm{hyp}} \nabla^{4} \overrightarrow{\mathrm{v}}_{\|} \\
\frac{\partial \psi}{\partial t}=\eta\left(j-j_{A}\right)+R[\psi, \Phi]-\frac{\partial \Phi}{\partial \phi} \\
\quad-\frac{\delta^{*} R}{\rho}\left[\psi, p_{e}\right]+\frac{\delta^{*}}{\rho} \frac{\partial p_{e}}{\partial \phi}+\eta_{\mathrm{hyp}} \nabla^{2} j \\
\frac{\partial \rho}{\partial t}=-\nabla \cdot\left(\rho \overrightarrow{\mathrm{v}}_{\mathrm{tot}}\right)+\nabla \cdot\left(D_{\perp} \nabla_{\perp} \rho\right)+S_{\rho}+D_{\mathrm{hyp}} \nabla^{4} \rho \\
\frac{\partial p}{\partial t}=-\overrightarrow{\mathrm{v}}_{E} \cdot \nabla p-\gamma p \nabla \cdot \overrightarrow{\mathrm{v}}_{E}+\nabla \cdot\left(\kappa_{\perp} \nabla_{\perp} T+\kappa_{\|} \nabla_{\|} T\right)+S_{T}
\end{gathered}
$$

where the density, temperature and current sources $S_{\rho}, S_{T}$ and $j_{A}$ have been introduced. The current source term $j_{A}$ also includes the time-dependent bootstrap current calculated using Sauter's formula [20]. The convective derivative, the parallel gradient, the perpendicular gradient, and the Poisson brackets are defined as

$$
\begin{gathered}
\frac{\mathrm{d}}{\mathrm{d} t}=\frac{\partial}{\partial t}+\overrightarrow{\mathrm{v}}_{E} \cdot \nabla, \\
\nabla_{\|}=\vec{b}[\vec{b} \cdot \nabla], \\
\nabla_{\perp}=\nabla-\nabla_{\|}, \\
{[\alpha, \beta]=\vec{e}_{\phi} \cdot(\nabla \alpha \times \nabla \beta),} \\
\vec{b}=\frac{1}{|B|} \vec{B} .
\end{gathered}
$$

Note that equations (3) and (4) can be reduced to scalar equations by projecting them in the poloidal and parallel directions, respectively, by applying the operators $\nabla \cdot\left[R \vec{e}_{\phi} \times()\right]$ and $\vec{b} \cdot()$. This reduced set of equations (without the diffusive transport terms and the diamagnetic terms) is equivalent to that derived in [21], where energy of the system is shown to be conserved at first order. 
The perpendicular mass and thermal diffusivities $D_{\perp}$ and $\kappa_{\perp}$ used in simulations are ad hoc coefficients with a well at the pedestal region to represent the $\mathrm{H}$-mode transport barrier. The profiles used for $D_{\perp}$ and $\kappa_{\perp}$ are discussed in section 2.2. A Spitzer-like resistivity $\eta=\eta_{o}\left(T_{\mathrm{e}} / T_{\mathrm{e}, o}\right)^{-3 / 2}$ is used, with $T_{\mathrm{e}, o}$ the electron temperature at the magnetic axis. Likewise, a temperature-dependent perpendicular viscosity is used: $\mu=\mu_{o}\left(T_{\mathrm{e}} / T_{\mathrm{e}, o}\right)^{-3 / 2}$. The Braginskii parallel thermal conductivity $\kappa_{\|}$is expressed as $\kappa_{\|}=\kappa_{\| o}\left(T / T_{o}\right)^{5 / 2}$. The ratio of specific heat is $\gamma=5 / 3$. Hyper-diffusive coefficients $\mu_{\text {hyp }}, \eta_{\text {hyp }}$ and $D_{\text {hyp }}$ are also used in these simulations.

The normalization of the equations is based on the magnetic permeability $\mu_{o}$ and the core density $\rho_{o}$, so that time is normalized to a near Alfven time $t=t_{\mathrm{SI}} / \sqrt{\mu_{o} \rho_{o}}$. For a deuterium plasma with particle density $n_{o}=6 \times 10^{19} \mathrm{~m}^{-3}$, a normalized time unit corresponds to approximately $0.5 \mu \mathrm{s}$. Naturally, current is normalized with $\mu_{o}$ and density with $\rho_{o}$. Pressure is also normalized with $\mu_{o}$, the diamagnetic frequency is normalised with time, as and $\delta^{*}=\delta_{\mathrm{SI}}^{*} \sqrt{\mu_{o} \rho_{o}}$ and the diffusive parameters are normalized as $\eta=\eta_{\mathrm{SI}} \sqrt{\rho_{o} / \mu_{o}}, \mu=\mu_{\mathrm{SI}} \sqrt{\mu_{o} / \rho_{o}}$, $D=D_{\mathrm{SI}} \sqrt{\mu_{o} \rho_{o}}$ and $\kappa=\kappa_{\mathrm{SI}} \sqrt{\mu_{o} / \rho_{o}}$.

The boundary of the computational domain in the SOL is a flux surface, on which Dirichlet boundary conditions (zero perturbation) are applied for all variables, except density and temperatures, for which Neumann conditions with null gradient are applied. At the divertor targets, sheath boundary conditions are used for the parallel velocity and the energy conduction, such that,

$$
\begin{aligned}
& \overrightarrow{\mathrm{V}}_{\mathrm{tot}} \cdot \vec{b}= \pm c_{s}= \pm \sqrt{\gamma T}, \\
& n T \overrightarrow{\mathrm{V}}_{\|}+\kappa_{\|} \nabla_{\|} T=\gamma_{s h} n T \overrightarrow{\mathrm{V}}_{\|} .
\end{aligned}
$$

where $\vec{b}$ is the unit vector parallel to the magnetic field. The density has free outflow boundary conditions at the target (no density reflection). In the private region, which is also bounded by a flux surface, Dirichlet conditions are used for all variables.

The 2D poloidal grid is composed with isoparametric cubic Bezier finite elements [4]. The finite element grid is aligned to equilibrium flux surfaces for the three regions of the core, the SOL and the private region. Alignment along open flux surfaces in the SOL is important in order to treat accurately the fast parallel transport of energy along magnetic field lines. The toroidal dimension is represented by a Fourier series.

The time stepping is done using the implicit CrankNicolson scheme, so that the size of time steps depends only on the time scale of the instabilities that are simulated. This implicit scheme results in a sparse system of equations, which is solved using a Generalized Minimal REsidual Solver (GMRES). The preconditioner for this iterative GMRES is obtained by solving independently each sub-matrix corresponding to different Fourier harmonics, which amounts to a block-Jacobi preconditioner. These sub-matrices are solved using the direct parallel sparse matrix solver PaStiX [22].

In order to allow the $n=0$ component of the $\vec{E} \times \vec{B}$ and parallel flows to evolve towards a stationary equilibrium, the

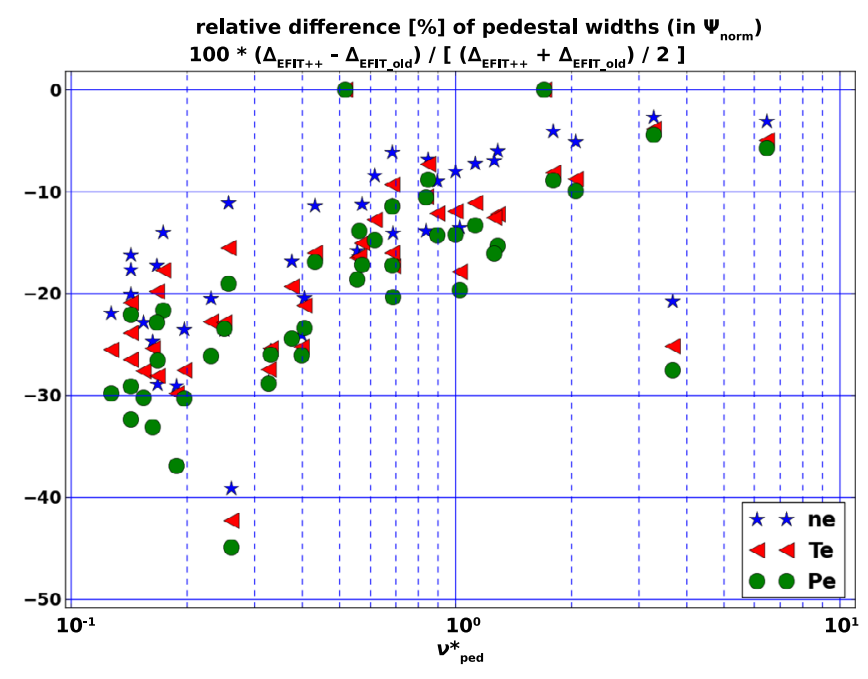

Figure 1. The pressure, polarimetry and MSE constraints in EFIT, together with allowing for pedestal current in the equilibrium reconstruction, have a strong effect on the mapping of the $n_{\mathrm{e}}$ and $T_{\mathrm{e}}$ profiles from the HRTS diagnostic. This plot shows the relative difference, in \%, between the standard EFIT and EFIT ++, for the pedestal width of the ne, Te and pe profiles. The $\psi$-mapped pedestal width can be $>30 \%$ smaller with EFIT ++ .

simulations are first run without toroidal modes, with only the equilibrium $n=0$, for $0.5 \mathrm{~ms}$. This allows the Bohm boundary conditions to diffuse into the SOL (at the zeroth time step, $\overrightarrow{\mathrm{v}}_{\|}$ is Mach-1 on the target, and zero inside the plasma, already at the nodes adjacent to the boundary)

\subsection{Simulating ELMs in JET}

The pulses used for the simulations are the same JET-ILW as in [14]: an $I_{\mathrm{P}}$ and a $\nu^{*}$ scan, with both low- and high- gas fuelling. In the experiments, the divertor heat-flux is observed to increase at lower pedestal collisionality, but one of the most notable effects is that of the gas fuelling: at similar $\nu_{\text {ped }}{ }^{*}$ values, a higher gas fuelling leads to higher ELM frequency, with lower ELM sizes and divertor heat-fluxes. In this previous study [14], the ELM energy losses were reproduced by the simulations, but the divertor heat-fluxes were lower than in experiments. The simulated ELMs were less intense, but lasted longer than in experiments (hence ELM energy losses were comparable). In the present study, more accurate pre-ELM equilibria were used, and larger toroidal resolutions as well as diamagnetic terms were included in the simulations.

In [14], the standard EFIT equilibria were used, with magnetic constraints only, in order to map the $n_{\mathrm{e}}$ and $T_{\mathrm{e}}$ profiles and reconstruct the JOREK Grad-Shafranov equilibria. A recent version of EFIT++/JEC2020 [23] was used to impose additional constraints (pressure, polarimetry, motional stark effect MSE) to the pre-ELM equilibria. The most important difference between the two versions of EFIT, with respect to ELM simulations presented here, is that the latter version allows for pedestal currents. This modifies the magnetic flux gradient in the pedestal region, such that when the highresolution Thomson scattering (HRTS) ne and Te profiles are mapped from real space onto the magnetic equilibrium, the 
resulting pedestal gradient width (in $\psi$-space) is modified. For these pulses it is found, as shown in figure 1, that the gradient width can be diminished as much as $40 \%$ for low collisionality pulses (i.e. those with the largest pedestal current). This effect was expected to have a strong influence on the discrepancy obtained in the first study of [14].

The pre-ELM $n_{\mathrm{e}}$ and $T_{\mathrm{e}}$ profiles used for the simulations are taken from the high-resolution Thomson scattering (HRTS) diagnostic, as described in [14, 24, 25]. In all simulations, $T i=T e$ is assumed, which will be discussed further in the context of low- and high-gas fuelling discharges. The pedestal current density is calculated according to the Sauter's bootstrap current model [20]. For high $\nu_{\text {ped }}^{*}$ discharges, it has been shown in [26, 27] that the Sauter formula [20] overestimates the bootstrap current, however in the present simulations this discrepancy was not accounted for and the Sauter formula was used for every discharge.

Note that the pre-ELM $n_{\mathrm{e}}, T_{\mathrm{e}}$ and current profiles are not taken directly from the EFIT ++ equilibrium itself, because EFIT ++ will find the optimal equilibrium that satisfies all various constraints (pressure, polarimetry, MSE and magnetics). This can result in output profiles that are slightly different from the input measured HRTS profiles. Therefore, the original pre-ELM profiles are used, with the consistent bootstrap current, and the Grad-Shafranov equilibrium is recalculated internally in JOREK, as described in [28]. Having equilibrium profiles and currents in coherence with the background $\psi$ map is important in nonlinear simulations, where the $\nabla p$ and $\vec{J} \times \vec{B}$ terms need to be properly balanced in the momentum equation (3).

In simulations below, the full diamagnetic effects are used, as well as multiple toroidal mode numbers, which was not the case in the previous study [14]. The new simulations were run with toroidal mode numbers $n=3,6,9,12,15$. A few simulations were run with the full toroidal spectrum from 1 to $15, n=1,2,3, \ldots, 15$ (although this was not possible for all pulses). All simulations were run with a resistivity level at a factor 10 above the Spitzer resistivity, which depends on the absolute temperature profile of each pulse. The viscosity level is $4.10^{-8} \mathrm{~kg} \cdot \mathrm{m}^{-1} \cdot \mathrm{s}^{-1}$ for all pulses. The parallel conductivity, which also depends on the absolute temperature for each pulse, was taken at a factor 4 above the ion Braginskii value (10x below the electron value). The factor 10 in resistivity results from numerical limitations: at low resistivity, diamagnetic effects are challenging due to strong diamagnetic currents obtained during the ELM crash. To avoid thin current layers below the grid resolution and below the electron gyroradius, hyper-resistivity is set to $\eta_{\text {hyp }}=10^{-13}$, which is approximately $10 \times \eta^{2}$ in the pedestal region, to ensure that MHD is not dominated by the numerical resistivity, rather than resistivity itself. The same value is used for the hyperviscosity $\mu_{\text {hyp }}$ and the hyper-diffusivity $D_{\text {hyp }}$.

The perpendicular diffusivity profiles $D_{\perp}$ and $\kappa_{\perp}$ are based on the initial density and temperature profiles in the edge region, with $D_{\perp} \sim 1 / \nabla \rho$ and $\kappa_{\perp} \sim 1 / \nabla T$, from $\psi_{n}=0.5$ up to outside the separatrix (at the bottom of the profiles). Hence, the perpendicular diffusive fluxes $D_{\perp} \nabla \rho$ and $\kappa_{\perp} \nabla T$ are constant in the pedestal, which ensures a rigid evolution of the density and temperature profiles. The value of $D_{\perp}$ and $\kappa_{\perp}$ is taken to be $5 \mathrm{~m}^{2} \mathrm{~s}^{-1}$ and $10^{-7}(\mathrm{~m} \cdot \mathrm{s})^{-1}$ respectively, at $\psi_{n}=0.8$, such that the diffusive evolution of the profiles is much longer than the typical time scale of an ELM $(\sim 1-5 \mathrm{~ms})$. Radially uniform sources $S_{\rho}$ and $S_{T}$ are used inside the separatrix to ensure that the (also radially uniform) perpendicular diffusive fluxes are balanced at equilibrium. This particular choice of diffusive profiles and sources was made because it is the most robust way to ensure that the profiles remain identical to the initial profiles, as measured by the HRTS diagnostic. Note that since the simulations are started with only $n=0$ (to allow for the formation of the stationary equilibrium), this ensures that the profiles do not deviate from the pre-ELM HRTS profiles in this period, before the $n>0$ toroidal modes are started, and the ELM crash begins. Eventually, integrated simulations should be run with more realistic transport and heating/fuelling models to determine what the exact values of diffusive parameters and sources should be in each JOREK simulations. However, such an inclusion of JOREK into integrated modelling structures would require a significant investment which, at this point, cannot be characterised as essential with respect to the peeling-ballooning instabilities themselves. In any case, there is no robust and reliable models for turbulent transport in the pedestal region in the presence of an $\mathrm{H}$-mode transport barrier.

The grid chosen for the simulations has elements radially concentrated in the pedestal region, so that the radial width of elements is $1.4 \mathrm{~mm}$ at the separatrix (at the midplane). The radial resolution in the core is kept high enough to avoid artificial ballooning instabilities with the highest mode number $n=15$. The poloidal distribution of elements is also controlled to ensure a uniform poloidal resolution, including at the X-point. The average poloidal width is $2.7 \mathrm{~cm}$. Since the time step of implicit schemes depends only on the nonlinear activity of the toroidal modes (i.e. not on the minimum element size of the 2D grid), the usual time step of the order of $0.5 \mu \mathrm{s}$, which is reduced up to $0.05 \mu \mathrm{s}$ in the most challenging cases. This means that a usual ELM simulation requires between 2000 and 10000 time steps.

\section{Simulation results for ILW pulses}

\subsection{Linear stability}

The first aspect of simulations to consider is the linear stability of the simulated discharges. It has now been established that, in JET-ILW type-I ELMy discharges with high gas fuelling, the linear stability of pre-ELM equilibria is typically incoherent with the linear ideal MHD stability theory, unless Nitrogen seeding is used [26, 29]. Linear MHD calculations of pre-ELM stability with the ELITE code $[30,31]$ shows that the ELM onset is typically well inside the stable region of the $j-\alpha$ stability diagram, where $j$ is the edge plasma density (responsible for destabilizing peeling modes), and $\alpha$ is the pedestal pressure gradient (responsible for destabilizing ballooning modes).

The linear stability of peeling-ballooning modes can also be evaluated using JOREK, including non-ideal MHD effects, such as resistivity, viscosity, diffusion and conductivity, NBI 


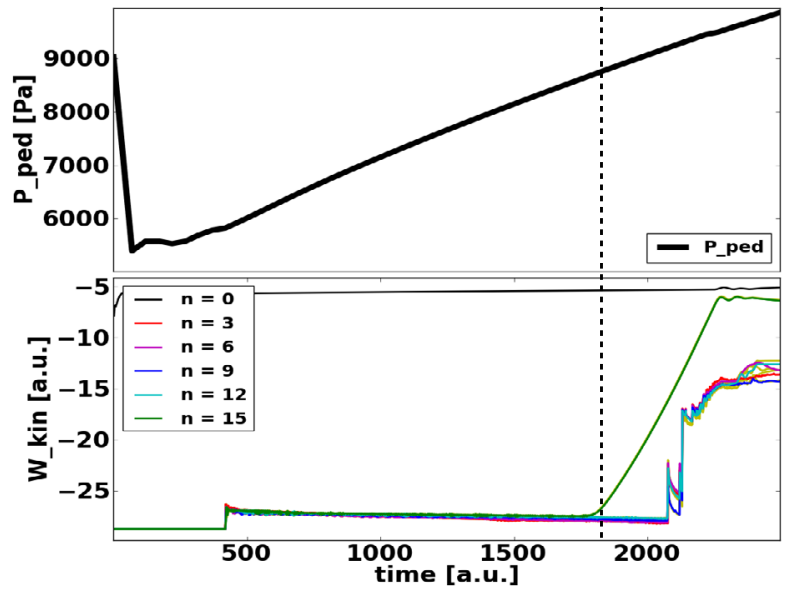

(a)

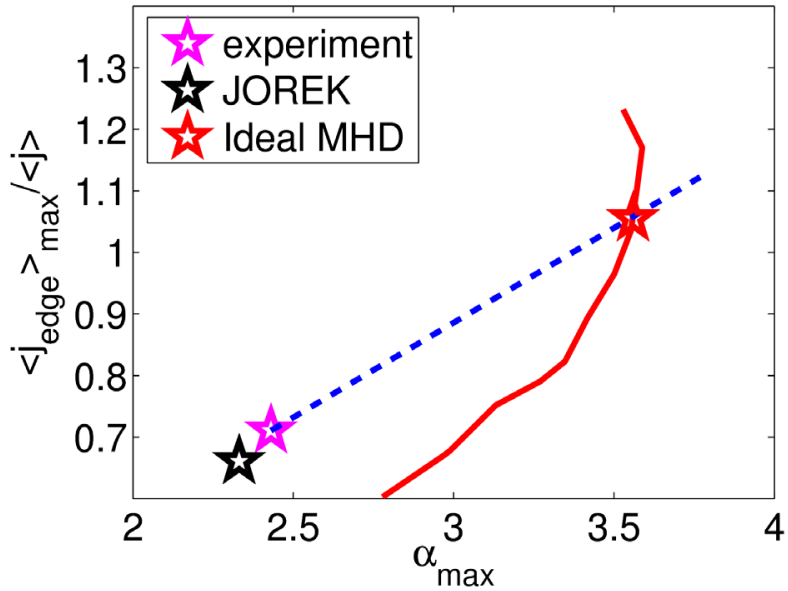

(b)

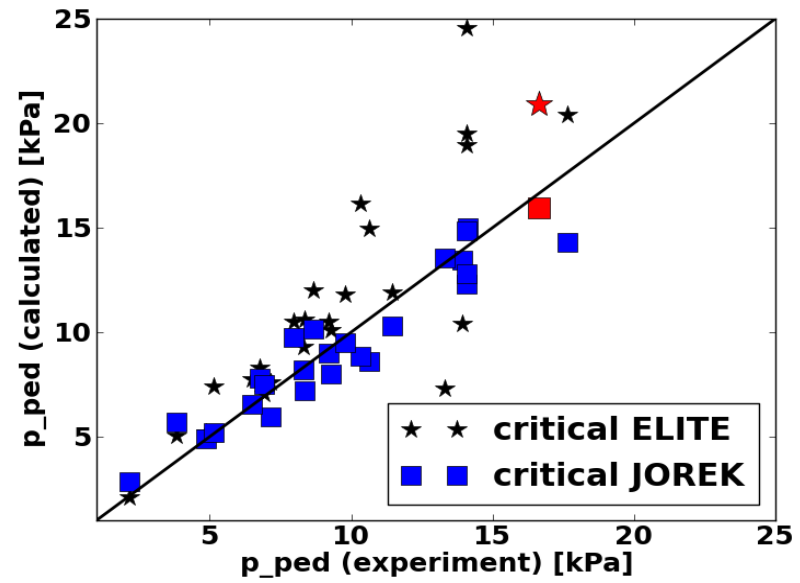

(c)

Figure 2. (a) A JOREK simulation starting from a stable $p_{\text {ped }}$ value. The top plot shows the evolution of $p_{\text {ped }}$, the bottom plot shows the evolution (on a logarithmic $y$-axis) of the kinetic energy of various ballooning modes. Keeping the pedestal width constant and increasing $p_{\text {ped }}$ progressively reveals the linear MHD threshold for peeling-ballooning modes (determined once a mode starts growing exponentially, here at $t \sim 1800$ ). The advantage over linear MHD codes is that non-ideal effects (resistivity, rotation etc) are easily included. The disadvantage is that it is numerically more expensive. $(b)$ An example is shown here, for pulse 83340, to illustrate how the JOREK code can, in some cases, obtain a better agreement with experiments than ideal MHD calculations. This is shown in the usual $j-\alpha$ diagram (the solid red line shows the stability boundary calculated by ELITE). The dashed blue line represents the coherent evolution of $\alpha_{\max }$ and $j_{\max }$, assuming that the bootstrap current evolves when $p_{\text {ped }}$ is increased (here according to the Sauter model). (c) This plot shows the linear calculations for all JET pulses included in this study, with the ELITE code (for ideal MHD), and with the JOREK code (for nonideal MHD). These calculations are compared to the experiments on the $x$-axis. Non-ideal MHD clearly provides a closer agreement with experiments. The red square/star is pulse 83340 .

toroidal rotation, and diamagnetic effects. This is achieved by starting simulations with a stable pedestal pressure, progressively increasing it through the stability threshold, as shown in figure 2(a). Once a coherent peeling-ballooning mode starts growing exponentially, the pedestal pressure level is used as the stability threshold. Note that this is done using a fixed pedestal width, and with density and temperature sources that result in the same $n_{\mathrm{e}}^{\text {ped }}$ and $T_{\mathrm{e}}^{\text {ped }}$ values as in the experiments once the experimental $p^{\text {ped }}$ is reached. Also, the bootstrap current (calculated using Sauter's formula [20]), is evolving according with the pedestal density and temperature profiles.

The results for linear ideal and non-ideal MHD calculations are shown in figure 2. Figure 2(a) shows how JOREK is used to evaluate the linear stability of the pedestal by progressively increasing $p^{\text {ped }}$ at fixed pedestal width. Figure $2(b)$ shows an example of the $j$ - $\alpha$ diagram for the JET-ILW pulse 83340, including the experimental pre-ELM equilibrium, the ideal MHD threshold (with the stability boundary) calculated by ELITE, and the non-ideal MHD threshold calculated by JOREK. Figure 2(c) shows the ideal and non-ideal calculations for all the ILW pulses considered in this study. This shows that in many cases the non-ideal MHD effects have a significant impact on the linear stability threshold. At this point it is not clear which of these particular effects has the most important impact on stability, and further work would be required to isolate each effect. It will be the scope of a future study, since this requires re-running each discharge 5 to 6 times (once for each isolated effect), which is not achievable on a short time scale.

This result demonstrates that JOREK could be used to improve our undestanding of MHD stability in JET-ILW 


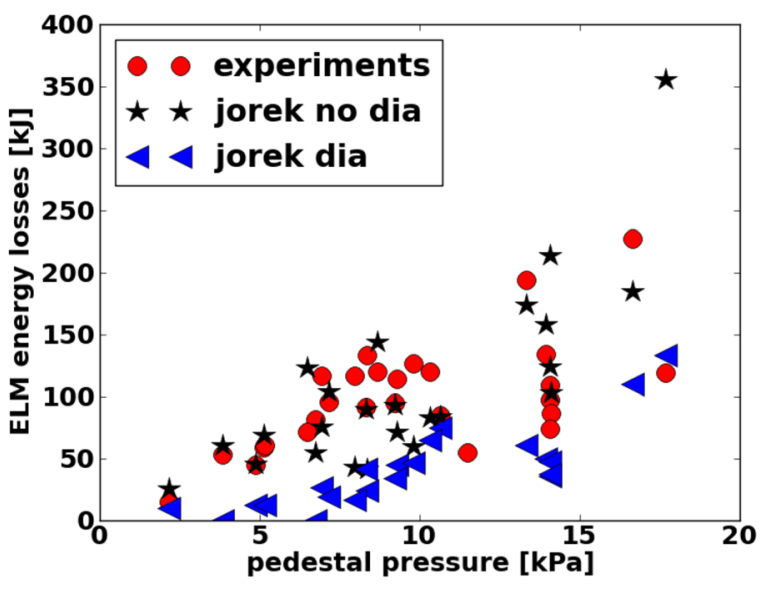

(a)

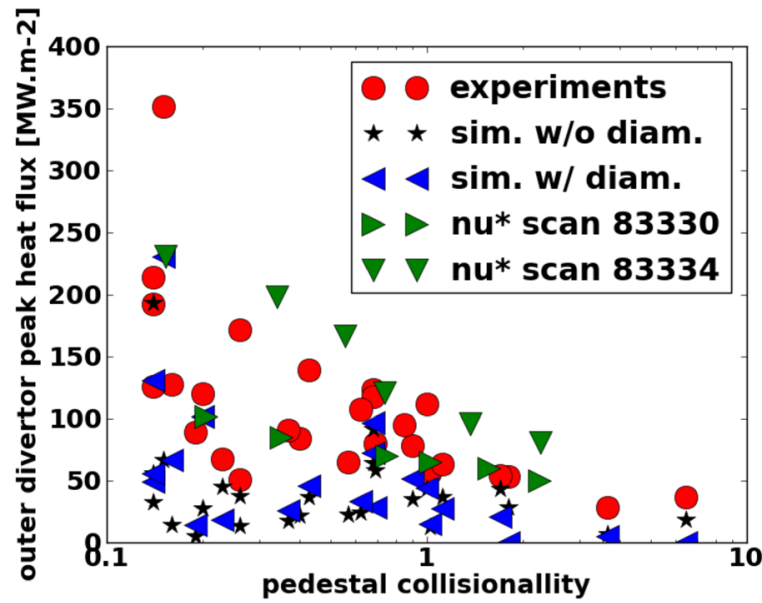

(b)

Figure 3. (a) This plot shows the total ELM energy losses as a function of $p_{\text {ped, }}$, for the experiments (red circles, calculated using pre- and post-ELM HRTS profiles), for the simulations without diamagnetic terms (black stars), and for simulations including diamagnetic terms (blue triangles). The diamagnetic effects stabilise the mode activity in the late phase of the ELM, leading to shorter bursts, and hence smaller ELM energy losses. (b) The peak heat flux on the outer divertor as a function of $\nu_{\text {ped }}^{*}$, for the experiments (red circles), for the simulations without diamagnetic terms (black stars), for simulations including diamagnetic terms (blue circles), and for two theoretical $\nu_{\text {ped }}^{*}$ scans based on pulses 83330 and 83334 (green triangles).

discharges. In particular, once the physics responsible for this correction on ideal MHD is isolated, JOREK could be used to provide a reinforced MHD stability calculation for peeling-ballooning modes in predictive models like EPED [32]. However, at this point a systematic inclusion of JOREK calculations into predictive models is strongly limited by the fact that such JOREK calculations (as shown in figure 2(a)) are numerically more expensive than ideal MHD calculations using dedicated codes like ELITE.

\subsection{ELM Size and divertor heat-fluxes}

As shown in figure 2, linear non-ideal MHD provides a better agreement with experiments than ideal-MHD for linear calculations. However, linear stability provides no estimate or guarantee concerning the size of the ELM crash itself. As contradictory as it may seem, while reasonable agreement is obtained for linear stability, the ELM crash itself is typically smaller in simulations. However, it is worth noting that the discharges which give the best agreement (in terms of ELM size and divertor heat-flux) are those closest to the linear ideal MHD threshold for PB-stability, which are typically the low gas fuelling discharges. In this section, the ELM simulations are obtained by starting from the exact pre-ELM equilibrium prescribed by experimental data. (i.e. not from a progressively increasing pressure gradient as described above.)

The ELM size calculated in JOREK simulations with diamagnetic terms are smaller than in the experiments by a factor $2-3$. This is a large decrease compared to the previous set of simulations without diamagnetic effects, as shown in figure 3(a). The experimental ELM size is determined using the pre- and post-ELM pressure profiles obtained from the HRTS diagnostic, as in [14]. It should be noted that the diamagnetic effects are dominating the non-linear phase of the ELM crash, rather than the linear burst and the initial filamentation of the plasma. Of course, linear stability is also affected, to the extent that some pulses that are PB-unstable without diamagnetic terms become stable with diamagnetic terms. In most cases however, with the diamagnetic terms, the initial burst of filaments is similar, but the duration of the instability is much shorter with diamagnetic terms. Without $\omega^{*}$ effects, filaments keep bursting through the separatrix until the excess pressure is evacuated from the pedestal. However with $\omega^{*}$ effects, at most one or two sets of filaments burst through the separatrix, after which the stabilising effect of the $\omega^{*}$ terms set an end to the ELM crash.

This aspect of the shortened ELM duration is certified by the dynamics of divertor heat fluxes. Figure $3(b)$ shows the peak heat-flux on the outer divertor for each pulse, as a function of pedestal collisionality $\nu_{\text {ped }}^{*}$. The experimental data is obtained using the Infra-Red camera diagnostic $[1,33]$, averaged over all type-I ELMs for each discharge. The divertor heat-flux is similar, for most pulses, to the previous simulations of [14]. The width of the outer divertor heat-flux $(10-12 \mathrm{~cm})$ is also similar to the experiments and to the previous simulations. The main effect of diamagnetic terms is to diminish the time duration of the ELM crashes. Note that at the lowest $\nu_{\text {ped }}^{*}$, there is a large span in peak divertor heat flux for the experimental points due to different gas fuelling levels, which has a strong influence on the ELM frequency, and therefore the ELM amplitude.

It should be pointed out that, of course, although simulations without diamagnetic effects give a better agreement with the experimentally measured ELM size, they are not more accurate than simulations without diamagnetic terms. On the contrary, the increased duration of the ballooning activity without diamagnetic terms should be seen as a physical artefact, even if it leads to the right ELM energy losses. In fact, the simulations without diamagnetic terms are more coherent in the sense that both the divertor heat-fluxes and the ELM size are smaller than in the experiments. Therefore, the issue is 


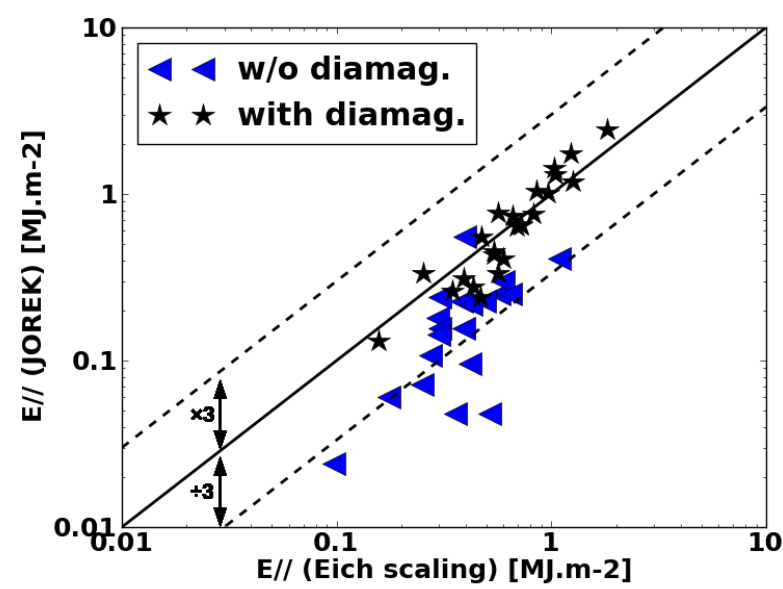

(a)

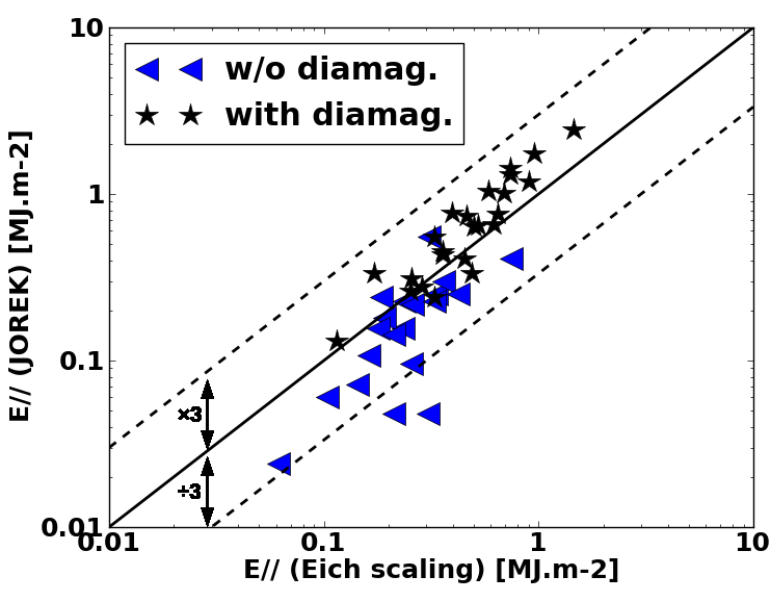

(b)

Figure 4. (a) The parallel energy arriving on the outer divertor can be compared against the Eich scaling law equation (16). The agreement is good provided the ELM size is comparable to the experiments. Hence, the best agreement is for the cases without diamagnetic terms.

Note that the Eich scaling used here is the exact regression $\varepsilon_{\|}=0.28 n_{\mathrm{e}, \text { ped }}^{0.75} T_{\mathrm{e}, \mathrm{ped}}^{0.98} \Delta W_{\mathrm{ELM}}^{0.52} R_{\mathrm{geo}}^{1.0}$ (b) The same plot, but using modified exponents, within the regression limits: $\varepsilon_{\|}=0.14 n_{\mathrm{e}, \mathrm{ped}}^{0.6} T_{\mathrm{e}, \mathrm{ped}}^{0.98} \Delta W_{\mathrm{ELM}}^{0.68} R_{\mathrm{geo}}^{1.0}$.

now to determine why the divertor heat-flux is smaller, since it represents the signature of up-stream MHD activity.

To address this question, we consider the cases that do have a good agreement with the experiments. In particular, two cases are relatively well reproduced, both in terms of divertor heat flux and energy losses: discharges 83330 and 83334 . These are low gas fuelling discharges with $\left[I_{\mathrm{P}}, B_{\mathrm{T}}, P_{\mathrm{NBI}}\right]=[2 \mathrm{MA}, 2 \mathrm{~T}, 20 \mathrm{MW}] \quad$ and $\left[I_{\mathrm{P}}, B_{\mathrm{T}}, P_{\mathrm{NBI}}\right]=[2.4 \mathrm{MA}, 2.4 \mathrm{~T}, 25 \mathrm{MW}]$ respectively. Using these two cases, a theoretical $\nu_{\text {ped }}^{*}$ scan was performed by varying the density and temperature levels at constant pressure. This ensures that the ideal MHD ballooning stability remains identical. In practice, this is equivalent to changing the normalisation of the non-ideal MHD parameters, such as resistivity, viscosity, thermal conductivity, and diamagnetic effects (as described in section 2.1). The result is in good agreement with the experiments, as shown in figure $3(b)$. This is important as it demonstrates that the parallel transport of energy along field lines is relatively well described by the Braginskii model.

Another quantity to consider for the validity of parallel transport with a Braginskii model is the parallel energy, as defined by Eich et al [34]:

$$
\varepsilon_{\|}(s)\left[\mathrm{MJ} \cdot \mathrm{m}^{-2}\right]=\int_{t_{\mathrm{ELM}}} \alpha_{B} q_{\|}(s, t) \mathrm{d} t
$$

where $s$ is the divertor (radial) coordinate, and $\alpha_{B}$ is the angle between magnetic field lines and the divertor target. The regression scaling obtained from the experimental data on multiple tokamak devices [34] shows a strong dependency on $p_{\text {ped }}$ and on the relative ELM size $\Delta W_{\text {ELM: }}$

$$
\begin{aligned}
\varepsilon_{\|}\left[\mathrm{MJ} \cdot \mathrm{m}^{-2}\right]= & \left.0.28\right|_{ \pm 0.14} \times n_{\mathrm{e}, \mathrm{ped}}^{0.75 \pm 0.15} \times T_{\mathrm{e}, \mathrm{ped}}^{0.98 \pm 0.1} \\
& \times \Delta W_{\mathrm{ELM}}^{0.52 \pm 0.16} \times R_{\mathrm{geo}}^{1.0 \pm 0.4}
\end{aligned}
$$

Both (15) and (16) can be calculated in simulations, and it is found that for cases with an ELM size that corresponds to the experiments, the Eich scaling is well reproduced. This is shown in figure 4, where the two formulas are compared, both for the cases with and without diamagnetic terms. The agreement is successful for the cases without diamagnetic terms, while there is a larger disparity for the cases with diamagnetic terms, due to the discrepancy in ELM size. However, the parallel heat transport model is the same regardless of the diamagnetic terms, and therefore, if future simulations with diamagnetic terms can be improved to match the experimental ELM size, this indicates that the Eich scaling should be recovered.

Hence, if the linear stability from the experiments is well reproduced, and the parallel energy transport is also coherent with experimental regressions (provided the ELM size is reproduced), then the next logical step would be to look at the physics model. In particular, since low-gas pulses like 83330 and 83334 are relatively well reproduced by JOREK, the neutrals model $[35,36]$ could be considered in future simulations. However, it should be noted that one effect from neutrals can already be eliminated, namely the outward density shift observed at high gas fuelling [37-39], since this is already taken into account in the pre-ELM equilibria. Hence, if the missing neutrals were responsible for the lower ELM energy losses in simulations, it would have to do with other effects. For example, at high gas fuelling, the temperature conducted along field lines would face larger parallel gradients in the divertor region due to stronger ionization and charge exchange near the target, which would increase the parallel energy fluxes $\kappa_{\|} \nabla_{\|} T$ in the divertor region and thus increase the rate at which the pedestal temperature is evacuated. Another example is that the shift observed between the pre-ELM $n_{\mathrm{e}}$ and $T_{\mathrm{e}}$ pedestal profiles at high fuelling should be verified for the ion temperature $T_{i}$ as well, which is not considered in present simulations, due to the time-resolution of the charge exchange diagnostic for these pulses (i.e. the $T_{i}$ profiles are ELM-averaged). Hence, if the $T_{i}$ profile shifts together with $n_{\mathrm{e}}$ (as opposed to having $T_{i}=T_{\mathrm{e}}$ ), the stability of the highfuelling discharges would be strongly affected. Such questions should be addressed in future studies. 


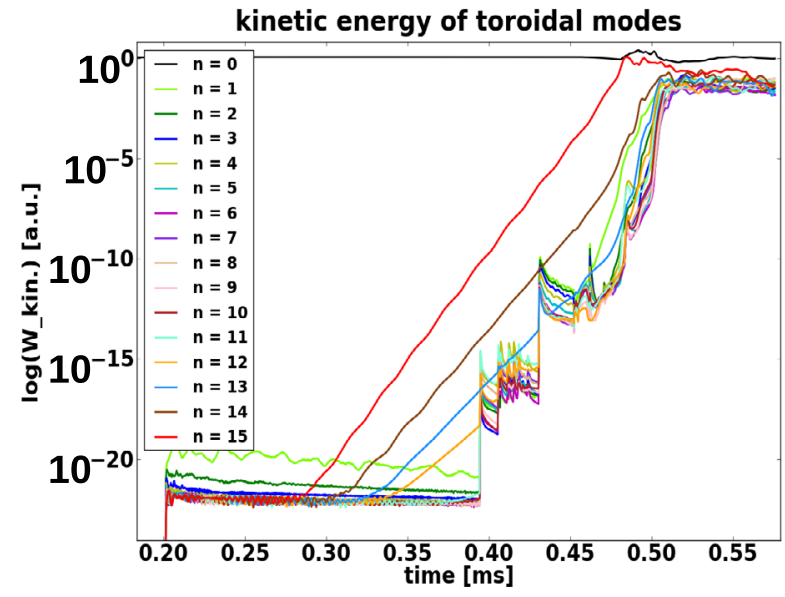

(a)

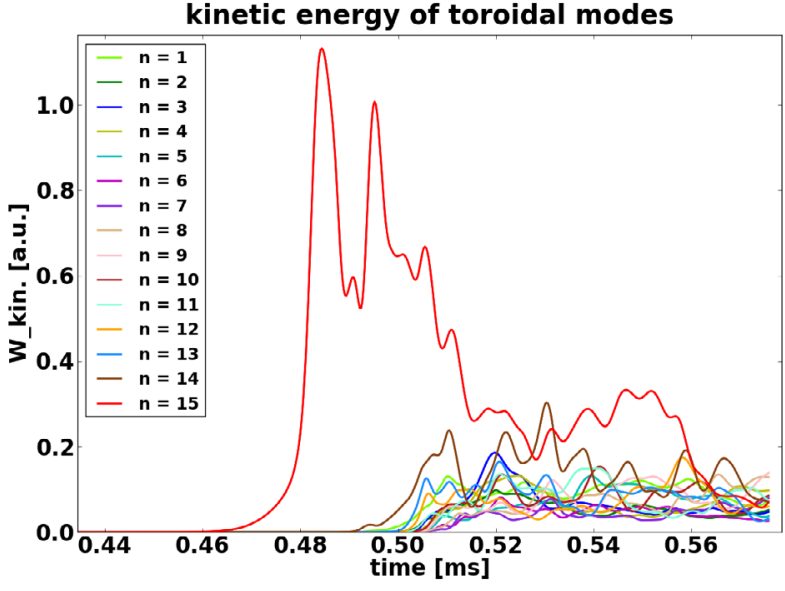

(b)

Figure 5. (a) The kinetic energy of the modes $n=1, \ldots, 15$ (in logarithmic scale) as a function of time. The mode $n=15$ has a slightly higher growth rate than the other modes. However, since the perturbations are started at a low numerical noise level, the exponential growth phase is long enough to cause a large delay between the arrival of $n=15$ and $n=14$ at equilibrium level. Therefore, by the time $n=14$ reaches equilirbium, $n=15$ has already produced a crash, which reduces $p_{\text {ped }}$, stabilising all other modes, and thus preventing any nonlinear coupling between modes. Note: the discontinuities observed at 0.40 and $0.43 \mathrm{~ms}$ are just a jump in numerical noise (those toroidal harmonics have not yet organised into peeling-ballooning structures). (b) The same plot, but not in logarithmic scale, and zoomed on the ELM crash itself, to show the obvious absence of any nonlinear coupling.

\subsection{Nonlinear stability and multi-ELM cycles}

There is, however, another aspect of simulations which should be addressed, because it could have a significant impact on ELM energy losses: the nonlinear dynamics of ELMs, and more particularly, the nonlinear stability of ELMs.

One of the peculiar aspects of the simulations presented in section 3.2 is the absence of nonlinear coupling between modes during the ELM crash. In most cases, a dominant mode number leads to the crash, while minor coupling with the other modes occurs only at the end of the ELM, once most of the energy has already been evacuated. In few cases, the coupling occurs earlier in the crash, but there is usually still one dominant mode number. It has been experimentally established that large type-I ELMs have a strong nonlinear component [40-42], notably for the main part of the crash. Thus, the quasi-linear aspect of ELMs in the simulations, even in the presence of diamagnetic terms, suggests that the set-up of simulations should be reconsidered.

In practice, any initial-value code like JOREK needs to start the non-axisymmetric perturbations as low numerical noise, which eventually organises into coherent peeling-ballooning structures, provided the equilibrium is unstable with respect to these instabilities. These peeling-ballooning structures will then grow exponentially, until they reach an amplitude large enough to perturb the background axisymmetric equilibrium, and thus create a crash. However, each toroidal Fourier harmonic, which represents an individual mode number, will grow at its individual rate. Since these growth rates depend on the mode number, one mode will grow more rapidly, and hence reach the equilibrium before all other modes. This is shown in figure 5 , which was run with the full spectrum of modes $n=1,2,3, \ldots, 15$. This simulation was performed to test whether one-to-one coupling between modes was required to obtain more nonlinear interactions between modes in the early phase of the ELM. However, the result is similar to the filtered spectrum $n=3,6,9,12,15$ : nonlinear coupling only occurs in the late phase of the ELM.

It is worth noting that the level of coupling in the early phase of the ELM strongly depends on the choice of MHD parameters, particularly resistivity and viscosity. In cases with higher resistivity and viscosity, nonlinear coupling in the early phase of the ELM is frequently observed, as in [17, 18]. However, the theoretical resistivity is set by the Spitzer value, and a higher viscous coefficient would stabilise the ballooning modes, which is the reason for the present choice of $\eta$ and $\mu$.

In experiments, the whole spectrum of modes is active at a certain level, not far below the equilibrium, and thus when an ELM arrives, all modes will crash together. It could even be assumed that nonlinear coupling is occurring before the ELM crash itself. In fact, it seems that the nonlinear coupling itself is responsible for the crash, as proposed in [43]. In order to simulate this, a state of marginal instability must be achieved, in which the various toroidal modes are fluctuating at equilibrium level without causing a crash. This is equivalent to going progressively from a stable to an unstable equilibrium, through the ELM onset threshold. In other words, it is equivalent to simulating multiple type-I ELM cycles.

JET pulse 83334 is started from a stable pedestal pressure, which is then restored using density fuelling and heating, a first small quasilinear crash is obtained, similar to those presented in figure 3 . Further increasing the pedestal pressure beyond this initial crash, strong nonlinear coupling is obtained, leading to a second large pedestal crash. This is represented in figure $6(a)$, which shows the evolution of the 


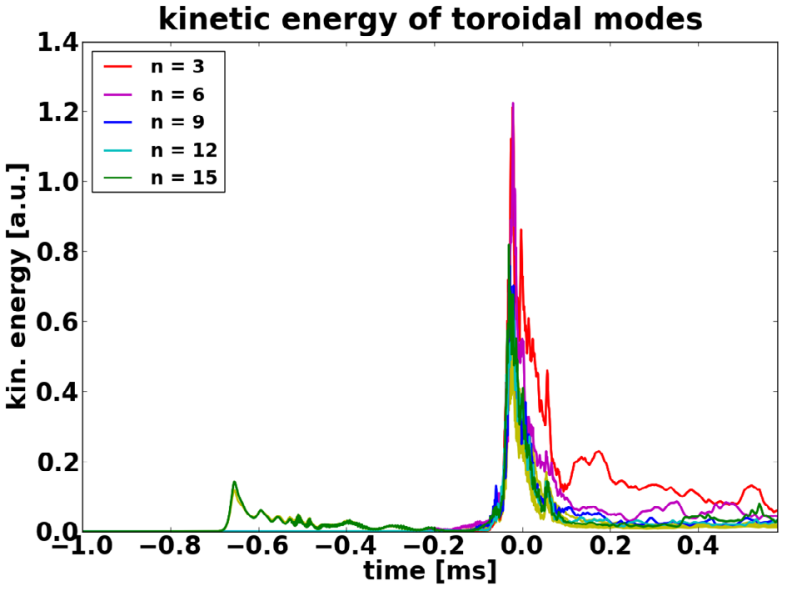

(a)
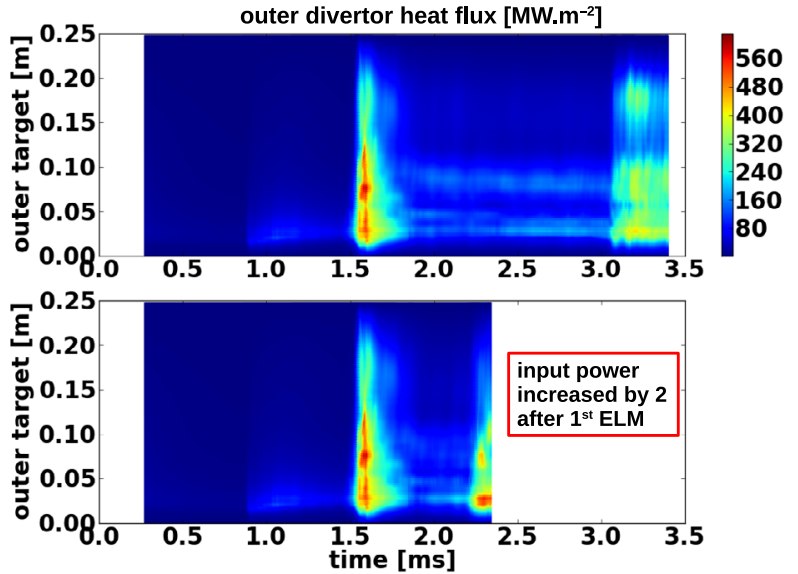

(b)

Figure 6. (a) The evolution of the kinetic energy of the modes for a simulation of pulse 83334, where density and temperture sources are used to progressively increase the pedestal pressure through the MHD stability threshold. Two clear events are observed: a quasilinear crash, at $-0.7 \mathrm{~ms}$, which is representative of the linear stability threshold, and a nonlinear crash, at $0.0 \mathrm{~ms}$, which represents a nonlinear MHD threshold. Although $p_{\text {ped }}$ is not much higher than for the first quasilinear crash $(\sim 10 \%)$, the nonlinear crash has a much larger amplitude. (b) After this large nonlinear crash, the $n_{\mathrm{e}}$ and $T_{\mathrm{e}}$ sources can be kept until a second ELM crash is found. The bottom case has sources a factor 2 higher than the top case, which results in an ELM period divided by 2. This is the signature of type-I ELMs: a frequency which increases with heating power.

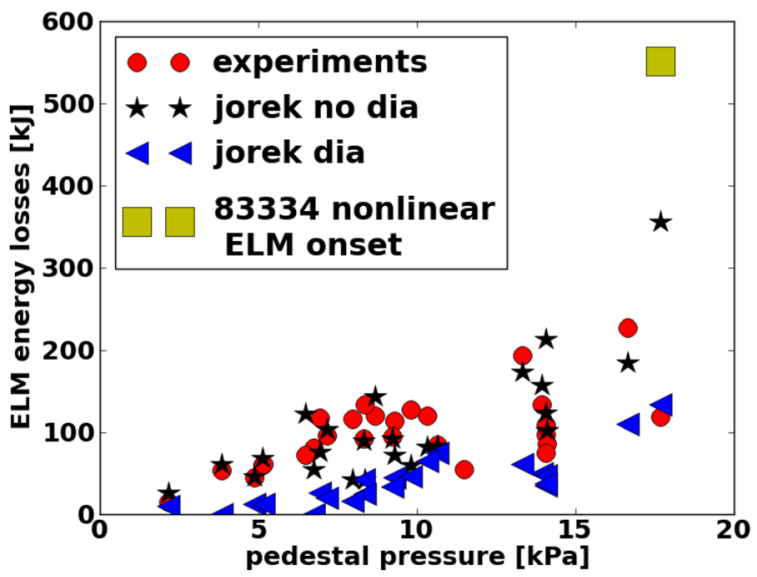

(a)

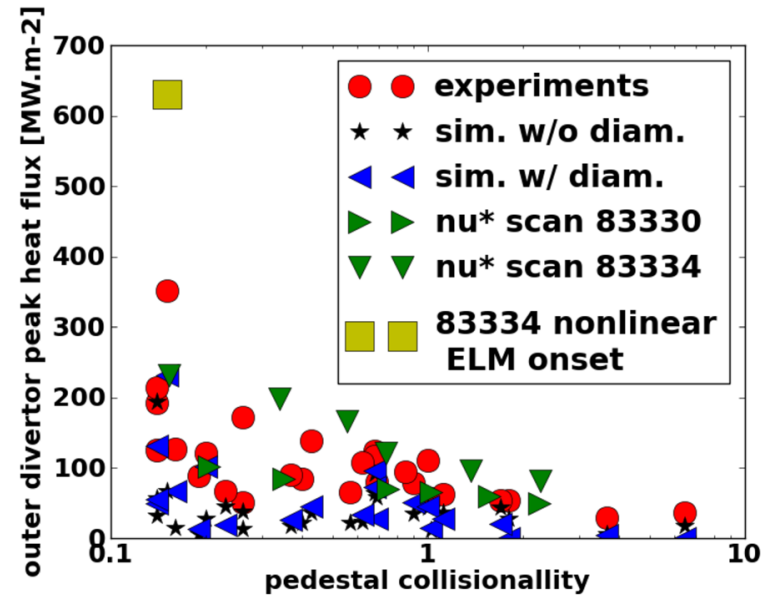

(b)

Figure 7. The same plot of the ELM size and the divertor peak heat-flux, as in figure 3, but including the nonlinear ELM crash of pulse 83334, detailed in figure 6. This demonstrates that the difference between linear and nonlinear ELM onsets can be significant.

peeling-ballooning modes in time. This second MHD event is clearly closer to a type-I ELM than the quasilinear events used in section 3.2.

Hence, it would be natural to attempt to use the same method for all pulses in order to obtain larger ELM crashes that would improve the comparisons in figure 3. However, there are fundamental issues with this approach. The first issue is that there is no guarantee that this second authentic type-I ELM will occur for all cases, and at a $p_{\text {ped }}$ level comparable to each corresponding experiment. For the above example, JET pulse 83334 , this type-I ELM occurs at a pedestal pressure $\sim 10 \%$ higher than in the experiments, and consequently has a larger ELM size and a higher divertor heat-flux. This is shown in figure 7.

The other issue concerns the reconstruction of the pedestal profiles in the pre-ELM phase. For case 83334, the width of the pedestal was kept constant, and only the pedestal height was increased, which is numerically the easier option. Ideally, the pedestal gradient should be prescribed at each time step, and for each radial point, by advanced gyro-kinetic simulations, or at least by pedestal transport models like the Kinetic Ballooning Mode (KBM) constraint used in EPED [32]. Such advanced integrated modelling should be explored in the future.

Finally, such simulations are numerically expensive: due to the high level of mode activity, time steps of the order of $0.05 \mu \mathrm{s}$ are required in the most nonlinear phase of the crash. At present, the prospect of such simulations on a quantitative basis, using 20-30 pulses, is unconceivable given the limited computational resources allocated to the JOREK simulations. Nevertheless, a complete validation of the JOREK code will require its ability to predict, on a quantitative basis, both the 
pre-ELM profiles and the ELM size, in agreement with experiments. Therefore, this step is unavoidable.

In theory, simulating the pre-ELM phase as well as the ELM itself is equivalent to simulating multiple-ELM cycles. In practice, this is not entirely true. This large nonlinear MHD event in figure 6(a), which we have attributed to a type-I ELM, occurs after a phase of low fluctuation of the toroidal modes. After this ELM crash, some substantial activity remains, particularly for the lowest mode number $n=3$. Using continued heating and density fuelling, the pedestal can be rebuilt until a second ELM crash occurs. Although the ELM frequency in simulations is higher than in experiments $(0.5-1.0 \mathrm{kHz})$, an encouraging characteristic of this second ELM crash is that it occurs after a period which directly depends on the level of heating and fuelling, just like type-I ELMs in experiments. This is shown in figure $6(b)$, where the bottom picture has heating and fuelling sources twice as large as the top picture, which reduces the inter-ELM period by two. However, the size of the second ELM also depends on the level of heating. This is thought to be due to the continued inter-ELM activity of the PB-modes, which make it difficult to achieve a large pedestal pressure gradient before the ELM occurs. However, the occurence of ELM precursors and inter-ELM fluctuations in both experiments and simulations $[14,28,44]$ suggests that multiple-ELM simulations should have some level of interELM MHD activity.

Of course, further work is yet needed to study the characteristics of such ELM cycles, and their comparability to type-I ELMs, but the power dependency provides an encouraging indication that these strongly nonlinear MHD events observed in simulations are similar to type-I ELMs.

\section{Conclusion}

\subsection{Summary}

In order to provide a quantitative validation of nonlinear MHD codes like JOREK, simulations need to be run for multiple experiments on multiple tokamak devices. This is a numerical and physical challenge that needs to be resolved in time to provide reliable predictions for future devices like JT-60SA, ITER and DEMO. These future tokamaks will require quantitative predictions of pedestal density and temperature levels, as well as inter-ELM and ELM divertor heat fluxes, in order to improve global confinement capabilities while preventing divertor erosion/melting in the planning of future experiments.

In this paper, we have shown the recent progress in the quantitative validation of the JOREK code using JET simulations. Reasonable agreement is found with the experiments for the linear stability of peeling-ballooning modes, which shows that non-ideal effects could improve predictive models like EPED that rely on ideal linear MHD codes. Regarding the pedestal collapse in simulations, diamagnetic effects are observed to play a strongly stabilising role, which reduces the total ELM energy losses. The peak heat-fluxes on the outer divertor are similar for cases with and without $\omega^{*}$, but the main difference comes from a damping of the nonlinear ballooning activity in the later phase of the ELM, which leads to a reduced loss in pedestal energy. The discharges which give the best agreement (in terms the ELM size and the divertor heat-flux) are those closest to the linear ideal MHD threshold for the peeling-ballooning stability, which are typically the low gas fuelling discharges.

A theoretical scan in collisionality has demonstrated that the experimental peak heat-flux dependency on $\nu_{\text {ped }}^{*}$ is recovered by the JOREK simulation. In addition, analysis of the divertor heat fluxes has shown that provided the ELM size is coherent with the experiments, the parallel energy arriving on the divertor target is consistent with the latest results from Eich et al [34]. It should be noted that this agreement was obtained even for cases where the simulated divertor peak heat-flux was lower than in the experiments, which can be misleading, and is due to the definition of $\varepsilon_{\|}$as the time-integrated peak heatflux. Nevertheless, such positive results regarding the parallel energy transport, from the pedestal to the target, are encouraging in the present context of plasma exhaust in future fusion devices, for which divertor protection is a major concern.

The remaining discrepancy in ELM size between simulations and experiments (still a factor 2, up to 3 in the worst cases) could be related to neutrals physics, since the largest differences occur at high gas fuelling. However, some tests were run to expose the fact that another crucial ingredient is missing, which could be as important as the neutrals physics, namely, the nonlinear stability of ELMs. It has been shown that when increasing the pedestal pressure from a stable to an unstable level, there are two distinct thresholds: a linear threshold and a nonlinear threshold. Although these two thresholds are not far from one another in terms of pedestal pressure $(\sim 10 \%)$, the resulting ELM dynamics and amplitude is entirely different. Simulations started with unstable pre-ELM profiles are almost systematically quasilinear (dominated by only one mode number), while simulations where the ELM emerges from background nonlinear fluctuations are, of course, nonlinear by nature, as is expected from the experimental evidence.

Such explosive instabilities will need to be addressed in the future, as it seems obvious that they change the picture of numerical ELM simulations and their quantitative validation against experiments. Such simulations are numerically more challenging, more expensive, and they require a more elaborate integrated methodology needed to provide the necessary information regarding anomalous diffusive transport due to turbulence, as well as heating and fuelling sources. Eventually, the complete validation of JOREK will only be achieved once simulations can predict, on a quantitative basis, the ELM energy losses, the divertor heat fluxes, but also the pre-ELM $p_{\text {ped }}$ level, which requires self-consistent simulations of multiple ELM cycles.

\subsection{Discussion for future improvements}

This conclusion leads to a number of open questions, which are worth mentioning here to finalise this discussion. In addition to the peeling-ballooning instabilities used to describe 
ELMs, small-scale turbulence simulations are needed for the inter-ELM regime. This is required not only for the evaluation of inter-ELM divertor heat-fluxes, but also for the prediction of pre-ELM pedestal pressure levels, which is determined broadly by how the pedestal profiles evolve until the ELM onset (determined by MHD stability). Turbulence codes like GS2, ORB5 [45, 46] (and many others not cited here) can evaluate the stability of KBMs in the pedestal, which are believed to regulate the pedestal pressure gradient during the inter-ELM phase [32, 47]. Although nonlinear MHD codes can evolve the inter-ELM pedestal profiles using ad hoc assumptions (e.g. fixed gradient or fixed width), in order to obtain reliable predictions for future devices, a coherent picture including turbulence is necessary.

In addition, both the inter-ELM pedestal evolution and the ELMs themselves share a common feature: the H-mode transport barrier. Significant progress has been achieved in recent years by nonlinear codes like HESEL, EMEDGE3D or CENTORI [7, 48, 49], and one of the main challenges of inter-ELM and ELM simulations at present is to determine whether a coherent description of the $\mathrm{H}$-mode transport barrier is required to obtain realistic and accurate evaluations of modes stability in the pedestal (both turbulence and PB modes). For example, in figure 6 , the pedestal width is kept constant, and the pedestal height is increased; in a more realistic picture, the pressure gradient should be clamped (e.g. by some micro-turbulence constraint like KBMs), and the width should be increased. However, in a fluid model which does not solve the H-mode transport barrier, this is not straightforward to obtain if modes fluctuate in the pre-ELM phase. In figure 6 , the method used works for an axisymmetric equilibrium with given perpendicular diffusivity profiles, but when modes are fluctuating in the pre-ELM phase, they modify the pedestal profiles constantly which makes it very challenging to constrain the profiles in any way. Hence, one of the most challenging questions that JOREK will need to address, in the near future, is whether simulations of multiple ELM cycles are possible without first resolving the $\mathrm{H}$-mode transport barrier in a coherent manner that includes small-scale turbulence.

\section{Acknowledgment}

This work has been carried out within the framework of the EUROfusion Consortium and has received funding from the Euratom research and training programme 2014-2018 under grant agreement No 633053, and from the RCUK Energy Programme (grant number EP/I501045). To obtain further information on the data and models underlying this paper please contact PublicationsManagerccfe.ac.uk. This work used the HELIOS supercomputer (IFERC-CSC), Japan, under the Broader Approach collaboration, implemented by Fusion for Energy and JAEA. The views and opinions expressed herein do not necessarily reflect those of the European Commission or the ITER Organization. The HEC ARCHER computer (UK), as part of the Plasma HEC Consortium EPSRC grant EP/L000237/1, and the MARCONI computer at CINECA in Italy, were also used.

\section{References}

[1] Eich T. et al 2009 J. Nucl. Mater. 760 390-1

[2] Eich T. et al 2013 Nucl. Fusion 53093031

[3] Huysmans G. 2007 Nucl. Fusion 47 659-66

[4] Czarny O. et al 2008 J. Comput. Phys. 227 7423-45

[5] Huijsmans G. et al 2013 Nucl. Fusion 53123023

[6] Dudson B. et al 2014 J. Plasma Phys. 81365810104

[7] Nielsen A. et al 2015 Phys. Lett. A 379 3097-101

[8] Fuhr G. et al 2008 Phys. Rev. Lett. 101195001

[9] Jardin S. et al 2005 Phys. Plasmas 12056101

[10] Sovinec C. et al 2004 J. Comput. Phys. 195355

[11] Todo Y. et al 2005 Phys. Plasmas 12012503

[12] Todo Y. et al 2006 Phys. Plasmas 13082503

[13] Khan R. et al 2007 Phys. Plasmas 14062302

[14] Pamela S. et al 2015 Plasma Phys. Control. Fusion 58014026

[15] Orain F. et al 2013 Phys. Plasmas 20102510

[16] Becoulet M. et al 2014 Phys. Rev. Lett. 113115001

[17] Hoelzl M. et al 2012 Phys. Plasmas 19082505

[18] Krebs I. et al 2013 Phys. Plasmas 20082506

[19] Strauss H.R. 1976 Phys. Fluids 19134

[20] Sauter O. et al 1999 Phys. Plasmas 67

[21] Strauss H.R. 1997 J. Plasma Phys. 57 83-7

[22] Henon P. et al 2008 Parallel Comput. 34 345-62

[23] Appel L. et al 2006 33rd European Physical Society (Rome, Italy, 29-23 June 2006) P2-184 (http://epsppd.epfl.ch/ Roma/pdf/P2_184.pdf)

[24] Frassinetti L. et al 2012 Rev. Sci. Instrum. 83013506

[25] Frassinetti L. et al 2015 Nucl. Fusion 55023007

[26] Maggi C. et al 2015 Nucl. Fusion 55113031

[27] Belli E. et al 2008 Plasma Phys. Control. Fusion 50095010

[28] Pamela S. et al 2011 Plasma Phys. Control. Fusion 53054014

[29] Saarelma S. et al 2015 Phys. Plasmas 22056115

[30] Wilson H. et al 2002 Phys. Plasmas 94

[31] Snyder P. et al 2002 Phys. Plasmas 95

[32] Snyder P. et al 2011 Nucl. Fusion 51103016

[33] Sieglin B. et al 2013 Plasma Phys. Control. Fusion 55124039

[34] Eich T. et al 2016 PSI Conf. (30 May 2016) (http://www. psi2016.enea.it/PSI_BOA/Abstracts/168_T_Eich_PSI22 TEich.pdf)

[35] Fil A. et al 2015 Phys. Plasmas 22062509

[36] Verbeek M. et al 2016 43rd EPS Conf. Proc. (Leuven, Belgium 4-8 July 2016) (http://ocs.ciemat.es/EPS2016PAP/pdf/ P5.058.pdf)

[37] Frassinetti L. et al 2017 Plasma Phys. Control. Fusion 59014014

[38] Stefanikova E. et al 2016 43rd European Physical Society Conf. Proc. (Leuven, Belgium, 4-8 July 2016) (http://ocs. ciemat.es/EPS2016PAP/pdf/O4.117.pdf)

[39] Dunne M.G et al 2017 Plasma Phys. Control. Fusion 59 014017

[40] Wenninger R. et al 2013 Nucl. Fusion 53113004

[41] Snyder P. et al 2005 Phys. Plasmas 12056115

[42] Kirk A. et al 2006 Plasma Phys. Control. Fusion 48 B433-41

[43] Wilson H. et al 2004 Phys. Rev. Lett. 92175006

[44] Becoulet M. et al 2016 Preprint: 2016 IAEA Fusion Energy Conf. (Kyoto, Japan, 17-22 October 2016) (https://nucleus. iaea.org/sites/fusionportal/Shared\%20Documents/FEC\%20 2016/fec2016-preprints/preprint0114.pdf)

[45] Kotschenreuther M. et al 1995 Comput. Phys. Commun. 88 128-40

[46] Jolliet S. et al 2007 Comput. Phys. Commun. 177409

[47] Saarelma S. et al 2017 Plasma Phys. Control. Fusion 59064001

[48] Chôné L. et al 2013 40th EPS Conf. (Helsinki, 1-5 July 2013) (http://ocs.ciemat.es/EPS2013PAP/pdf/P2.173.pdf)

[49] Thyagaraja A. et al 2010 Phys. Plasmas 17042507

[50] Litaudon X. 2017 Overview of the JET results in support to ITER Nucl. Fusion (https://doi.org/10.1088/1741-4326/ aa5e28) 